\title{
A GENERALIZED WEYL EQUIDISTRIBUTION THEOREM FOR OPERATORS, WITH APPLICATIONS
}

\author{
BY \\ J. R. BLUM( $\left.{ }^{1}\right)$ AND V. J. MIZEL( $\left.{ }^{2}\right)$
}

\begin{abstract}
The present paper is motivated by the observation that Weyl's equidistribution theorem for real sequences on a bounded interval can be formulated in a way which is also meaningful for sequences of selfadjoint operators on a Hilbert space.

We shall provide general results on weak convergence of operator measures which yield this version of Weyl's theorem as a corollary. Further, by combining the above results with the von Neumann ergodic theorem, we will obtain a Cesàro convergence property, equivalently, an "ergodic theorem", which is valid for all (projectionvalued) spectral measures whose support is in a bounded interval, as well as for the more general class of positive operator-valued measures. Within the same circle of ideas we deduce a convergence property which completely characterizes those spectral measures associated with "strongly mixing" unitary transformations. The final sections are devoted to applications of the preceding results in the study of complexvalued Borel measures as well as to an extension of our results to summability methods other than Cesàro convergence. In particular, we obtain a complete characterization, in purely measure theoretic terms, of those complex measures on a bounded interval whose Fourier-Stieltjes coefficients converge to zero.
\end{abstract}

1. Introduction. The present paper is motivated by the observation that Weyl's equidistribution theorem for real sequences on a bounded interval can be formulated in a way which is also meaningful for sequences of selfadjoint operators on a Hilbert space. Namely, if $\left\{x_{j}\right\}$ is a sequence on $[0,2 \pi]$ then the (equivalent) assertions

$$
\lim _{n \rightarrow \infty} \frac{1}{n} \sum_{j=0}^{n-1} \exp \left(i m x_{j}\right)=0, \quad m= \pm 1, \pm 2, \ldots
$$

and

$$
\lim _{n \rightarrow \infty} \frac{1}{n} \sum_{j<n: x_{j} \in[a, b]} 1=(b-a) / 2 \pi \text { for all }[a, b] \subset[0,2 \pi]
$$

can be regarded as assertions about the (selfadjoint) multiplication operators $\left\{x_{j}\right\}$ on the space of complex numbers. In fact, by writing (E2) in the equivalent form

$$
\lim _{n \rightarrow \infty} \frac{1}{n} \sum_{j=0}^{n-1} \chi_{[a, b]}\left(x_{j}\right)=(b-a) / 2 \pi \text { for all }[a, b] \subset[0,2 \pi]
$$

Received by the editors January 20, 1971 and, in revised form, June 30, 1971.

AMS 1969 subject classifications. Primary 4730, 2850, 6030; Secondary 2813.

Key words and phrases. Weak convergence of operator measures, ergodic theorem for spectral measures, convergence methods, generalized Riemann-Lebesgue Lemma.

(1) Research supported in part by NSF Grant No. GP-8290.

(2) Research supported in part by NSF Grant No. GP-11679. 
it is clear that the usual functional calculus makes equations (E1) and (E2') meaningful even when $\left\{x_{j}\right\}$ denotes a sequence of selfadjoint operators on a Hilbert space. The question then arises as to whether, with an appropriate notion of convergence, the equations remain equivalent in this new context.

We shall provide general results on weak convergence of operator measures which yield this version of Weyl's theorem as a corollary. Further, by combining the above results with the von Neumann ergodic theorem, we will obtain a Cesàro convergence property, equivalently, an "ergodic theorem", which is valid for all (projection-valued) spectral measures whose support is in a bounded interval, as well as for the more general class of positive operator-valued measures. Within the same circle of ideas we deduce a convergence property which completely characterizes those spectral measures associated with "strongly mixing" unitary transformations. The final sections are devoted to applications of the preceding results in the study of complex-valued Borel measures as well as to an extension of our results to summability methods other than Cesàro convergence. In particular, we obtain a complete characterization, in purely measure theoretic terms, of those complex measures on a bounded interval whose Fourier-Stieltjes coefficients converge to zero.

2. Weak convergence for operator measures. Hereafter $H$ will denote a separable Hilbert space and $[H]$ will denote the space of bounded linear transformations on $H$. The symbol $v_{n} \stackrel{c}{\rightarrow} v$ will denote the statement $(1 / n) \sum_{j=0}^{n-1} v_{j} \rightarrow v$, convergence being taken in the relevant linear topological space containing the $v_{n}$ and $v$. For simplicity of presentation all our considerations will be taken relative to the interval $[0,2 \pi]$ whenever possible. The scaling needed to apply our results to other bounded real intervals will be obvious. For any real interval $J, \mathscr{B}(J)$ denotes the class of Borel subsets of $J$. Finally, $Q$ denotes the subset of $[0,2 \pi)$ consisting of rational multiples of $\pi$.

Let us recall that a sequence $\left\{\nu_{n}\right\}$ of real or complex-valued (hence totally finite) Borel measures on an interval $J \subset(-\infty, \infty)$ is said to converge weakly to a measure $v_{0}$ provided

$$
\int_{J} f d \nu_{n} \rightarrow \int_{J} f d \nu_{0} \quad \forall \text { bounded continuous real } f \text {. }
$$

Moreover when the $\left\{\nu_{n}\right\}$ are given as equibounded it suffices for the existence of a weak limit $\nu_{0}$ that [9]

$$
\int_{J} \exp (i(2 \pi m /|J|) \theta) \nu_{n}(d \theta) \rightarrow \text { a limit, } \quad m=0, \pm 1, \pm 2, \ldots,
$$

where $|J|$ denotes the length of $J$.

Our first result is, in effect, the assertion in the case of the weak operator topology that certain alternate formulations of weak convergence of measures which are equivalent to (w0) for positive real Borel measures remain equivalent for positive operator Borel measures. 
THEOREM 1. Let $H$ be a separable Hilbert space and let $\left\{F_{k}\right\}_{k \geqq 1}$ be a sequence of weakly countably additive Borel measures on $[0,2 \pi)$ whose values are positive operators on $H$. Suppose that for each $x, y \in H$ the sequence of complex measures defined by

$$
\nu_{x, y}^{k}(B)=\left(F_{k}(B) x, y\right), \quad B \in \mathscr{B}[0,2 \pi), k \geqq 1,
$$

has equibounded variation

$$
\operatorname{Var} \nu_{x, y}^{k} \leqq K_{x, y}, \quad k \geqq 1 .
$$

Then the following conditions are equivalent, convergence being taken in weak operator topology:

$$
\int e^{i m \theta} F_{k}(d \theta) \rightarrow C_{m}, \quad m=0, \pm 1, \pm 2, \ldots,
$$

(w2) there exists a unique weakly countably additive positive-operator-valued Borel measure $F_{0}$ such that $F_{k}(B) \rightarrow F_{0}(B)$ whenever $B \in \mathscr{B}[0,2 \pi)$ is an $F_{0}$-continuity set, i.e. $F_{0}(\bar{B}-\not{B})=0$.

Moreover $F_{0}$ and the sequence $\left\{C_{m}\right\}$ are related as follows:

$$
C_{m}=\int e^{i m \theta} F_{0}(d \theta), \quad m=0, \pm 1, \pm 2, \ldots
$$

REMARKS. 1. It will be seen in the course of the proof that, for each positiveoperator-valued measure, the collection of continuity sets includes every interval whose boundary is disjoint from the countable collection of one-point atoms for this measure. The same arguments also yield this conclusion for other ("signed") operator-valued measures. However, the implication $(w 1) \Rightarrow(w 2)$ is generally false for sequences of "signed" operator measures, even in one dimension.

2. The equiboundedness of variation prescribed in $\left(^{*}\right)$ is, by the uniform boundedness principle, equivalent to the condition $\left\|F_{k}(B)\right\| \leqq K$ for $B \in \mathscr{B}[0,2 \pi), k \geqq 1$.

3. The arguments given below can be extended to the case in which $[0,2 \pi)$ is replaced by a second countable locally compact abelian group $\Lambda$ and $\left\{e^{i m \theta}\right\}_{m \geqq 1}$ is replaced by $\tilde{\Lambda}$, the character group of $\Lambda$. Equation (w1) is then replaced by the assertion that $\int \tilde{\lambda}(\lambda) F_{k}(d \lambda) \rightarrow C_{\tilde{\lambda}}$, uniformly on compact subsets of $\tilde{\Lambda}$. (See [9], for example.)

Proof. From $\left({ }^{*}\right)$ we obtain as in Remark 2 the appraisal

$$
\operatorname{Var} \nu_{x, y}^{k} \leqq 4 K\|x\|\|y\|, \quad k \geqq 1 .
$$

We show now that (w1) implies (w2). Given $x, y \in H$ we have

$$
\int e^{i m \theta} v_{x, y}^{k}(d \theta) \rightarrow\left(C_{m} x, y\right), \quad m=0, \pm 1, \pm 2, \ldots,
$$

which, in view of (1) and ( $\left(\mathrm{w}^{\prime}\right)$, ensures the existence of a unique complex Borel measure $\nu_{x, y}^{0}$ to which the sequence $\left\{\nu_{x, y}^{k}\right\}_{k \geqq 1}$ converges weakly. It follows that

$$
\int e^{i m \theta} v_{x, y}^{0}(d \theta)=\left(C_{m} x, y\right), \quad m=0, \pm 1, \pm 2, \ldots,
$$


and

$$
\operatorname{Var} \nu_{x, y}^{0} \leqq 4 K\|x\|\|y\|,
$$

while the positiveness of the measures $\left\{\nu_{x, x}^{k}\right\}$ ensures that each $\nu_{x, x}^{0}$ is a positive measure. Obviously $\nu_{x, y}^{0}$ is also bilinear as a function of $x, y$. Thus for each $B \in \mathscr{B}[0,2 \pi)$ we may write

$$
\nu_{x, y}^{0}(B)=\left(F_{0}(B) x, y\right)
$$

where $F_{0}(B) \in[H]$, by $(3)$, and $F_{0}(B)$ is positive since $\nu_{x, x}^{0}(B) \geqq 0$ for all $x \in H$. The weak -ountable aciditivity of this set function $F_{0}$ is clear.

Now let $S \subset H$ be a denumerable dense subset of $H$ which we can take to be closed under addition and under scalar multiplication by $i$. Given $x \in S$ the weak convergence of the positive measures $\nu_{x, x}^{k}$ to $\nu_{x, x}^{0}$ implies by Alexandroff's theorem [2] that $\nu_{x, x}^{k}(B) \rightarrow \nu_{x, x}^{0}(B)$ for each $\nu_{x, x}^{0}$-continuity set $B: \nu_{x, x}^{0}(\bar{B}-B)=0$. Now for each positive measure $\mu$ on $[0,2 \pi)$ there exists a denumerable subset $D_{\mu}$ such that all intervals whose boundary is in the complement of $D_{\mu}$ are $\mu$-continuity sets. Thus it follows from the denumerability of $S$ that the collection of Borel sets which are simultaneous $\nu_{x, x}^{0}$-continuity sets for all $x \in S$ includes every interval whose boundary is in the complement of a fixed countable set. We denote the collection of simultaneous $\nu_{x, x}^{0}$-continuity sets by $\mathscr{B}_{1}$ so that

$$
\nu_{x, x}^{k}(B) \rightarrow \nu_{x, x}^{0}(B) \text { for all } x \in S, B \in \mathscr{B}_{1} .
$$

Observe next that our hypotheses on $S$ ensure that each of the complex Borel measures $\left\{\nu_{x, y}^{0}\right\}_{x, y \in S}$ can be obtained by polarization from the positive Borel measures $\left\{\nu_{x, x}^{0}\right\}_{x \in S}$ :

$$
\nu_{x, y}^{0}=\frac{1}{4}\left[\nu_{x+y, x+y}^{0}-\nu_{x-y, x-y}^{0}+i \nu_{x+i y, x+i y}^{0}-i \nu_{x-i y, x-i y}^{0}\right] .
$$

This follows from the analogous decomposition for the measures $\left\{v_{x, y}^{k}\right\}_{x, y \in S}$. Clearly (6) implies that the sets $B \in \mathscr{B}_{1}$ are continuity sets for the complex measures $\left\{\nu_{x, y}^{0}\right\}_{x, y \in S}$ as well as for the $\left\{\nu_{x, x}^{0}\right\}_{x \in S}$, and that

$$
\nu_{x, y}^{k}(B) \rightarrow \nu_{x, y}^{0}(B) \text { for all } x, y \in S, B \in \mathscr{B}_{1} .
$$

We proceed to show that (7) also holds for arbitrary $x, y \in H$. Utilizing (1) and (3) we can write

$$
\begin{aligned}
\left|\nu_{x, y}^{k}(B)-v_{x^{\prime}, y^{\prime}}^{k}(B)\right| & =\left|\left(F_{k}(B)\left(x-x^{\prime}\right), y\right)+\left(F_{k}(B) x^{\prime}, y-y^{\prime}\right)\right| \\
& \leqq K\left(\left\|x-x^{\prime}\right\|\|y\|+\left\|y-y^{\prime}\right\|\|x\|\right), \quad k \geqq 0, B \in \mathscr{B}[0,2 \pi] .
\end{aligned}
$$

It follows from this that for $x, y \in H$ and $\left\{x_{i}\right\},\left\{y_{i}\right\} \in S$ satisfying $x_{i} \rightarrow x, y_{i} \rightarrow y$ one has, uniformly in $k$,

$$
\operatorname{Var}\left(\nu_{x, y}^{k}-\nu_{x_{i}, y_{i}}^{k}\right) \rightarrow 0, \quad k=1,2, \ldots,
$$

and similarly $\operatorname{Var}\left(\nu_{x, y}^{0}-\nu_{x_{i}, y_{i}}^{0}\right) \rightarrow 0$. This ensures the validity of (7) for arbitrary $x, y \in H$ and hence the validity of (w2). 
To prove the converse we note that the arguments of the preceding paragraph imply that for any operator measure $F$ the $F$-continuity sets consist of precisely those sets which are simultaneous continuity sets for some countable family of measures of the form $\{(F x, x)\}_{x \in S}$, and that this collection contains all intervals whose endpoints are disjoint from a fixed countable subset of $[0,2 \pi)$. From this it follows, in particular, that each function $e^{i m \theta}$, being a bounded continuous function on $[0,2 \pi]$, is a uniform limit of simple functions based on the class $\mathscr{B}_{1}$ of $F_{0^{-}}$ continuity sets, thus, given $x, y \in H$ we have

$$
\begin{aligned}
\mid \int e^{i m \theta}\left(F_{k}(d \theta) x, y\right) & -\int e^{i m \theta}\left(F_{0}(d \theta) x, y\right) \mid \\
\leqq & \left|\int\left(e^{i m \theta}-s(\theta)\right) \nu_{x, y}^{k}(d \theta)\right|+\left|\int\left(e^{i m \theta}-s(\theta)\right) \nu_{x, y}^{0}(d \theta)\right| \\
& +\left|\int s(\theta) \nu_{x, y}^{k}(d \theta)-\int s(\theta) \nu_{x, y}^{0}(d \theta)\right|
\end{aligned}
$$

where $s$ may be taken as a simple function based on $\mathscr{B}_{1}$. Here the terms on the right side can be made arbitrarily small by choosing $s$ sufficiently near $e^{i m \theta}$ in the uniform norm and by choosing $k$ sufficiently large. This completes the proof.

Our second result shows that a similar extension is available in the strong operator topology-provided that the $\left\{F_{k}\right\}$ have values which are mutually commuting.

THEOREM 2. Let $H$ and the $\left\{F_{k}\right\}$ be as in Theorem 1 and suppose in addition that the $\left\{F_{k}\right\}$ commute:

$$
F_{k}(B) F_{k^{\prime}}\left(B^{\prime}\right)=F_{k^{\prime}}\left(B^{\prime}\right) F_{k}(B), \quad k, k^{\prime} \geqq 1, B, B^{\prime} \in \mathscr{B}[0,2 \pi) .
$$

Then the conditions (w1) and (w2) are equivalent for convergence in strong operator topology.

Proof. By a theorem of von Neumann on commuting families of operators on a separable space $[11$, p. 360$]$, all operators in the family $\left\{F_{k}(B)\right\}_{k \geqq 1, B \in \mathscr{B}[0,2 \pi)}$ are functions of some fixed bounded hermitian operator $A$. Thus if $E$ denotes the spectral measure for $A$ and $J$ denotes the smallest closed interval supporting $E$, then there exists for each pair $k, B, k \geqq 1, B \in \mathscr{B}[0,2 \pi)$, an element $f^{k}(B, \cdot) \in \mathscr{L}^{2}(E, J)$ such that

$$
F_{k}(B)=\int_{J} f^{k}(B, \sigma) E(d \sigma), \quad k \geqq 1, B \in \mathscr{B}[0,2 \pi) .
$$

By $(1), f^{k}(B, \cdot)$ is actually in $\mathscr{L}^{\infty}(E, J)$, and

$$
\left\|f^{k}(B, \cdot)\right\|_{L^{\infty}} \leqq K, \quad k \geqq 1, B \in \mathscr{B}[0,2 \pi) .
$$

Moreover the positivity of the operator $F_{k}(B)$ implies that $f^{k}(B, \cdot) \geqq 0 E$-a.e. We now observe that there exists a version $\left\{\hat{f}^{k}(B, \cdot)\right\}$ of the family $\left\{f^{k}(B, \cdot)\right\}$ possessing the following properties: 
(i) $0 \leqq \hat{f}^{k}(B, \sigma) \leqq K$ for all $\sigma \in J, k \geqq 1$,

(ii) $\hat{f}^{k}(\cdot, \sigma)$ is a measure on $\mathscr{B}[0,2 \pi)$ for all $\sigma \in J, k \geqq 1$,

(iii) $\hat{f}^{k}(B, \cdot)$ is $\mathscr{B}(J)$-measurable for all $B \in \mathscr{B}[0,2 \pi), k \geqq 1$.

This follows from the existence of a lifting for the Banach space $L^{\infty}(E, J)([6]$, [3], [5]), that is a mapping from each class $\hat{f} \in L^{\infty}(E, J)$ to a function $\hat{f} \in \mathscr{L}^{\infty}(E, J)$ such that $\dot{f} \geqq 0$ implies $\hat{f} \geqq 0$ everywhere, and $\dot{f}_{n} \rightarrow \dot{f}_{0}$ in $L^{\infty}$ if and only if $\hat{f}_{n} \rightarrow \hat{f}_{0}$ uniformly on $J$. If we replace each $f^{k}(B, \cdot)$ by the equivalent function $f^{k}(B, \cdot)$ then (i) and (iii) are obvious, while the countable additivity of $\hat{f}^{k}(\cdot, \sigma)$ required in (ii) follows from the fact that the positive operator measures $F_{k}$ are actually countably additive and hence the classes $f^{k}(B)$ in $L^{\infty}(E, J)$ are countably additive in the norm topology.

Since $\hat{f}^{k}$ is, roughly speaking, a transition probability we can write (w1) in the following form [8]:

$$
\int e^{i m \theta} F_{k}(d \theta)=\int\left[\int e^{i m \theta} \hat{f}^{k}(d \theta, \sigma)\right] E(d \sigma) \rightarrow C_{m}, \quad m=0, \pm 1, \ldots,
$$

where $C_{m}$, as a limit of functions of $A$, is itself a function of $A: C_{m}=\int c_{m}(\sigma) E(d \sigma)$. From the strong convergence

$$
\int\left[\int e^{i m \theta} \hat{f}^{k}(d \theta, \sigma)\right] E(d \sigma) \rightarrow \int c_{m}(\sigma) E(d \sigma)
$$

it follows that the integrands converge in $L^{2}(E, J)$ :

$$
\int e^{i m \theta} \hat{f}^{k}(d \theta, \cdot) \rightarrow c_{m}(\cdot) \text { in } L^{2}(E, J), \quad m=0, \pm 1, \pm 2, \ldots
$$

Equation (10) implies that every subsequence of $\{k\}$ possesses a subsequence, $\left\{k_{i}\right\}$ say, such that

$$
\int e^{i m \theta} \hat{f}^{k_{i}}(d \theta, \sigma) \rightarrow c_{m}(\sigma) \quad E \text {-a.e., } \quad m=0, \pm 1, \pm 2, \ldots
$$

Now the equiboundedness of the positive measures $\hat{f}^{k}(\cdot, \sigma)$ together with (11) ensures that for each $\sigma \in J$, excluding an $E$-null set $N$, there exists a positive measure $f^{0}(\cdot, \sigma)$ to which the $\hat{f}^{k_{i}}(\cdot, \sigma)$ converge weakly:

$$
\hat{f}^{k_{i}}(\cdot, \sigma) \rightarrow f^{0}(\cdot, \sigma) \text { weakly, for } \sigma \notin N .
$$

In particular, $f^{\circ}(\cdot, \sigma) \leqq K$ for $\sigma \notin N$, and $c_{m}(\sigma)=\int e^{i m \theta} f^{0}(d \theta, \sigma), \sigma \notin N$, while by Alexandroff's theorem [2] we have for each $\sigma \notin N$

$$
\hat{f}^{k_{i}}(B, \sigma) \rightarrow f^{0}(B, \sigma)
$$

whenever $B$ is an $f^{\circ}(\cdot, \sigma)$-continuity set: $f^{\circ}(\bar{B}-\stackrel{B}{B}, \sigma)=0$. We wish next to define a set function $F_{0}$ on $\mathscr{B}[0,2 \pi)$ as follows:

$$
F_{0}(B)=\int f^{\circ}(B, \sigma) E(d \sigma), \quad B \in \mathscr{B}[0,2 \pi) .
$$


Hence we must verify that $f^{\circ}(B, \cdot)$ is $E$-summable for all $B \in \mathscr{B}[0,2 \pi)$. It will suffice by (i) to show that $f^{0}([0, t), \cdot)$ is $E$-summable for all $t \in(0,2 \pi]$. Now by (12) it follows that [12]

$$
\begin{aligned}
f^{0}([0, t), \sigma) & \leqq \liminf \hat{f}^{k_{i}}([0, t), \sigma) \leqq \lim \sup \hat{f}^{k_{i}}([0, t], \sigma) \\
& \leqq f^{0}([0, t], \sigma), \quad t \in(0,2 \pi), \sigma \notin N .
\end{aligned}
$$

Hence for $\sigma \notin N$

$$
f^{0}([0, t), \sigma)=\lim f^{k_{i}}([0, t], \sigma)
$$

whenever $(\sigma, t)$ is not a point of one of the sets

$$
\begin{aligned}
A & =\left\{(\sigma, t): \lim \inf \hat{f}^{k_{i}}([0, t), \sigma) \neq \lim \sup \hat{f}^{k_{t}}([0, t], \sigma), \sigma \notin N\right\}, \\
A^{\prime} & =\left\{(\sigma, t): \lim _{n \rightarrow \infty} \lim \sup \hat{f}^{k_{i}}\left(\left[0, t-\frac{1}{n}\right), \sigma\right)<\lim \inf \hat{f}^{k_{i}}([0, t), \sigma), \sigma \notin N\right\} .
\end{aligned}
$$

Now $A$ and $A^{\prime}$ are $\mathscr{B}[0,2 \pi) \times \mathscr{B}(J)$-measurable since the positive functions $\hat{f}^{k}([0, t), \sigma)$ are measurable in $\sigma$ and monotone and left continuous in $t$, hence jointly measurable. Moreover (12) implies that for each $\sigma \notin N$ the subset $A \cup A^{\prime}$ meets $[0,2 \pi) \times \sigma$ in a subset which is denumerable, hence of Lebesgue measure zero.

It now follows by Fubini's theorem and (14) that $f^{\circ}([0, t), \cdot)$ is $E$-summable for Lebesgue almost all $t$. Using the monotonicity and left continuity in $t$ we see that $f^{0}([0, t), \cdot)$ is in fact summable for all $t \in[0,2 \pi)$, and after modification on the null set $[0,2 \pi) \times N$ is in fact jointly measurable. We will henceforth suppose the latter modification to have been made.

By the definition of $F_{0}$ and the monotone convergence theorem it follows that $F_{0}$ is strongly countably additive and that the collection of $F_{0}$-continuity sets is given by

$$
\mathscr{B}_{0}=\left\{B: f^{0}(\bar{B}-\not{B}, \sigma)=0 E \text {-a.e. }\right\} \subset \mathscr{B}[0,2 \pi) .
$$

Moreover by (12) and the dominated convergence theorem we deduce that every subsequence of $\{k\}$ possesses a subsequence $\left\{k_{i}\right\}$ such

$$
F_{k_{i}}(B)=\int f^{k_{i}}(B, \sigma) E(d \sigma) \rightarrow \int f^{0}(B, \sigma) E(d \sigma)=F_{0}(B), \quad \text { for } B \in \mathscr{B}_{0},
$$

convergence being in the strong operator topology. From this we conclude that in fact

$$
F_{k}(B) \rightarrow F_{0}(B) \text { for } B \in \mathscr{B}_{0},
$$

which completes the proof that (w1) implies (w2).

For the converse we observe that for each $m$ the function $g_{m}(\theta)=e^{i m \theta}$ is bounded and uniformly continuous on $[0,2 \pi)$. Denote by $\nu_{m, \sigma}=f^{0}(\cdot, \sigma) \circ g_{m}^{-1}$ the positive Borel measure on the unit circumference in the complex plane associated to 
$f^{0}(\cdot, \sigma)$ by $g_{m}$. The image under $g_{m}^{-1}$ of each $\nu_{m, \sigma}$-continuity set is an $f^{0}(\cdot, \sigma)$ continuity set:

$$
\nu_{m, \sigma}\left(\bar{C}-C^{\ell}\right)=0 \Rightarrow f^{\circ}\left(\left(g_{m}^{-1}(C)\right)^{-}-\left(g_{m}^{-1}(C)\right)^{\circ}, \sigma\right)=0 .
$$

Moreover the $\nu_{m, \sigma}$-continuity sets include all arcs on the unit circumference whose endpoints lie in the complement of the countable subset $P_{m, \sigma}=g_{m}\left(A_{\sigma}\right), A_{\sigma}$ being the set of atoms of $f^{0}(\cdot, \sigma)$ on $[0,2 \pi)$. It will now suffice to show that the collection

$$
\mathscr{B}_{m}=\left\{B: B=g_{m}^{-1}(C), \nu_{m, \sigma}\left(\bar{C}-C^{\prime}\right)=0 \text { E-a.e. in } \sigma\right\}
$$

has the property that $g_{m}$ is in the uniform closure of the class of simple functions based on $\mathscr{B}_{m}$. For by (17), (13) and the dominated convergence theorem one has

$$
\begin{aligned}
\int s(\theta) F_{k_{i}}(d \theta) & =\int\left[\int s(\theta) \hat{f}^{k_{i}}(d \theta, \sigma)\right] E(d \sigma) \\
& \rightarrow \int\left[\int s(\theta) f^{0}(d \theta, \sigma)\right] E(d \sigma)=\int s(\theta) F_{0}(d \theta)
\end{aligned}
$$

whenever $s$ is a simple function based on $\mathscr{B}_{m}$, while by (1)

$$
\begin{aligned}
\left\|\int\left(g_{m}(\theta)-s(\theta)\right) F_{k}(d \theta)\right\| \leqq\left\|g_{m}-s\right\|_{\infty}\left\|F_{k}([0,2 \pi))\right\| \leqq K\left\|g_{m}-s\right\|_{\infty}, & \\
& k=0,1, \ldots .
\end{aligned}
$$

Combining these appraisals one obtains

$$
\int e^{i m \theta} F_{k_{i}}(d \theta) \rightarrow \int e^{i m \theta} F_{0}(d \theta)
$$

Since every subsequence of $\{k\}$ possesses a subsequence $\left\{k_{i}\right\}$ for which (19) holds, (w2) is then proved. Finally, we shall show that $\mathscr{B}_{m}$ has the requisite property by noting that the $\mathscr{B}[0,2 \pi) \times \mathscr{B}(J)$-measurability of $f^{0}([0, t), \sigma)$ implies that the set $\left\{(t, \sigma): t \in P_{m, \sigma}\right\}$ is $\mathscr{B}[0,2 \pi) \times \mathscr{B}(J)$-measurable and hence an $l \times E$-null set, $l$ being normalized Lebesgue measure on $[0,2 \pi)$. It follows by Fubini's theorem that there exists a subset $T_{m}$ of total $l$-measure on the unit circumference such that $z \in T_{m}$ $\Rightarrow z \notin P_{m, \sigma} E$-a.e. in $\sigma$. This clearly implies that $\mathscr{B}_{m}$ has the required property, even if one restricts attention to simple functions based on arcs, and thus completes the proof.

We are now able to deduce the desired results on equidistribution of operators as corollaries.

Corollary 1. Let $\left\{S_{k}\right\}$ be a sequence of positive selfadjoint operators on the separable Hilbert space $H$ with spectra contained in $[0,2 \pi)$. Let the associated unitary operators and spectral measures be denoted by $U_{k}=e^{i S_{k}}$ and $\left\{E_{k}\right\}$, respectively. Then the following conditions are equivalent, convergence being taken in the weak operator topology:

(w1) $U_{k}^{m} \stackrel{c}{\rightarrow} C_{m}, m=0, \pm 1, \pm 2, \ldots$,

(w2) there exists a positive-operator-valued measure $F_{0}$ on $[0,2 \pi)$ such that 
$E_{k}(B) \stackrel{c}{\rightarrow} F_{0}(B)$ for each $F_{0}$-continuity set $B$.

If the $S_{k}$ are mutually permutable then (w1) and (w2) are also equivalent for convergence in the strong operator topology.

Proof. The corollary follows from Theorems 1 and 2 provided we take $F_{k}$ $=(1 / k) \sum_{1}^{k} E_{k}, k=1,2, \ldots$

Corollary 2. Let $H$ and $\left\{S_{k}\right\}$ be as in Corollary 1. Then the following conditions are equivalent, convergence being taken in the weak operator topology or strong operator topology according to whether the $\left\{S_{k}\right\}$ commute:

(w1) $U_{k}^{m} \stackrel{c}{\rightarrow} 0, m= \pm 1, \pm 2, \ldots$,

(w2) $E_{k}(J) \stackrel{c}{\rightarrow} l(J) I$ for each interval $J \subset[0,2 \pi)$, where l denotes normalized Lebesgue measure on $[0,2 \pi)$.

This last result suggests that the desired extension of Weyl's notion of equidistribution to operators $\left\{S_{k}\right\}$ with spectrum in $[0,2 \pi)$ consists in the requirement

$$
\frac{1}{n} \sum_{k=0}^{n-1} E_{k}([a, b))=\frac{1}{n} \sum_{k=0}^{n-1} \chi_{[a, b)}\left(S_{k}\right) \rightarrow \frac{b-a}{2 \pi} I,
$$

whenever $[a, b) \subset[0,2 \pi)$. In this context Weyl's criterion becomes the formula

$$
\frac{1}{n} \sum_{k=0}^{n-1} U_{k}^{m} \rightarrow 0, \quad m= \pm 1, \pm 2, \ldots
$$

It should be noted that there is an analogous notion of equidistribution for positive operators $\left\{S_{k}\right\}$ which are not uniformly bounded. Here we must replace $E_{k}([a, b))$ by $\sum_{r=0}^{\infty} E_{k}([a, b)+r 2 \pi)=E_{k}\left([a, b)^{\wedge}\right)$, where $[a, b)^{\wedge}$ is the periodic extension of $[a, b)$ by $2 \pi$. This corresponds to the application of the usual Weyl equidistribution theorem to the fractional parts of a sequence of real numbers.

3. An ergodic theorem for operator measures. Our next result is a direct outgrowth of the above ideas together with the von Neumann ergodic theorem. Let us denote by $\left\{\tau_{k}\right\}_{k \geqq 1}$ the following set of transformations of the Borel sets in $[0,2 \pi)$ :

$$
\tau_{k}(A)=\bigcup_{j=0}^{k-1}\left(\frac{1}{k} A+\frac{2 \pi j}{k}\right), \quad \text { where } \frac{1}{k} A+\frac{2 \pi j}{k}=\left\{t\left|t=t^{\prime}\right| k+\frac{2 \pi j}{k}, t^{\prime} \in A\right\} .
$$

It is then clear that for any Borel measure $F$ on $[0,2 \pi)$ the set function $F \circ \tau_{k}$ defined by $\left(F \circ \tau_{k}\right)(A)=F\left(\tau_{k}(A)\right)$ is also a Borel measure on $[0,2 \pi)$.

THEOREM 3. Let $E$ be a spectral measure on $[0,2 \pi)$ having as its values projections in a not necessarily separable Hilbert space $H$. Then the following relation holds for every Borel set $B$ whose boundary contains only points incommensurable with $\pi$, convergence being taken in the strong operator topology:

$$
E \circ \tau_{k}(B) \stackrel{c}{\longrightarrow} l(B)(I-E(Q))+\sum_{s=1}^{\infty} l_{s}(B) E\left(B_{s}\right),
$$


where

$$
B_{1}=\{0\}, \quad B_{s}=\bigcup_{0<j<s:(j, s)=1}\left\{\frac{2 \pi j}{s}\right\}, \quad s>1,
$$

and where l denotes normalized Lebesgue measure, with $l_{s}, s \geqq 1$, denoting the discrete measure of total mass 1 equally distributed among the points $\{2 \pi j / s\}_{0 \leqq j<s}$.

Proof. By the von Neumann ergodic theorem applied to the unitary operator $U=\int e^{i \theta} d E$ we have

$$
U^{k}=\int e^{i k \theta} d E \stackrel{c}{\longrightarrow} E(\{0\})=\int \chi_{\{0\}}(\theta) d E,
$$

where convergence is in the strong operator topology. Moreover the functional calculus for $U$ supplies an entire family of analogous relations. Namely,

$$
U^{k m}=\int e^{i k m \theta} d E \stackrel{c}{\longrightarrow} E\left(A_{m}\right)=\int \chi_{A_{m}}(\theta) d E, \quad m=0, \pm 1, \pm 2, \ldots
$$

where $A_{0}=[0,2 \pi), A_{m}=\bigcup_{j=0}^{|m|-1}\{2 \pi j /|m|\}$, convergence again being in the strong operator topology. This follows from the formula

$$
\frac{1}{n} \sum_{k=0}^{n-1} e^{i k m \theta} \rightarrow \chi_{A_{m}}(\theta), \quad \theta \in[0,2 \pi), \quad m=0, \pm 1, \pm 2, \ldots,
$$

since the convergence in (24) is obviously bounded. We may write (24) as

$$
\int e^{i m \varphi} \mu_{n, \theta}(d \varphi) \rightarrow \chi_{A_{m}}(\theta), \quad \theta \in[0,2 \pi), \quad m=0, \pm 1, \pm 2, \ldots
$$

where $\mu_{n, \theta}$ denotes the discrete measure assigning mass $1 / n$ to each of the points $\{k \theta(\bmod 2 \pi)\}_{0 \leqq k \leqq n-1}$. It follows from $(25)$ by $\left(w 0^{\prime}\right)$ that for each $\theta$ the positive measures $\left\{\mu_{n, \theta}\right\}$ converge weakly to a positive measure $\mu_{\theta}$ which satisfies

$$
\int e^{i m \varphi} \mu_{\theta}(d \varphi)=\chi_{A_{m}}(\theta), \quad \theta \in[0,2 \pi), \quad m=0, \pm 1, \pm 2, \ldots,
$$

and

$$
\mu_{n, \theta}(B)=\frac{1}{n} \sum_{k=0}^{n-1} \chi_{B}((k \theta)) \rightarrow \mu_{\theta}(B) \text { for all } \mu_{\theta} \text {-continuity sets } B
$$

where $(t) \in[0,2 \pi)$ and $(k \theta)=k \theta \bmod 2 \pi$. By the use of $(26)$ it is easily verified that the measures $\mu_{\theta}, \theta \in[0,2 \pi)$, are as follows:

$$
\begin{aligned}
\mu_{\theta} & =l, & & \theta / \pi \text { irrational, } \\
& =l & &
\end{aligned}
$$

In particular, all Borel sets whose boundary is in the complement of the set $Q$ of rational multiples of $\pi$ are simultaneous $\mu_{\theta}$-continuity sets for all $\theta$. Moreover, by 
writing (27) in the form $\chi_{B}((k \theta)) \stackrel{c}{\rightarrow} \mu_{\theta}(B)$, we deduce that, in the strong operator topology,

$$
\int \chi_{B}((k \theta)) E(d \theta) \stackrel{c}{\longrightarrow} \int \mu_{\theta}(B) E(d \theta)
$$

for all $B$ which are simultaneous $\mu_{\theta}$-continuity sets. This last equation can be simplified by noting that $\chi_{B}((k \theta))=\chi_{\tau_{k}(B)}(\theta)$. Thus we obtain by (27)

$$
\left(E \circ \tau_{k}\right)(B)=\int \chi_{\tau_{k}(B)}(\theta) E(d \theta) \stackrel{c}{\longrightarrow} \int_{[0,2 \pi)-Q} l(B) d E+\sum_{s=1}^{\infty} l_{s}(B) E\left(B_{s}\right),
$$

as claimed.

REMARK 4. If $H$ is taken to be separable then Theorem 3 is essentially a direct corollary of Theorem 2. We simply observe that equation (23) above can be rewritten as

$$
U^{k m}=\int e^{i m \theta} d E \circ \tau_{k} \stackrel{c}{\longrightarrow} E\left(A_{m}\right)
$$

after which the existence of a weakly countably additive measure $F_{0}$ such that $E \circ \tau_{k}(B) \stackrel{c}{\rightarrow} F_{0}(B)$ for $F_{0}$-continuity sets $B$ is assured. The actual calculation of $F_{0}$ is as above.

The above theorem can be extended to positive operator measures as well. As shown by Naimark [7] each positive operator measure has a dilation to a spectral measure on a larger Hilbert space. Thus if $F$ is a positive operator measure on $[0,2 \pi)$ with $F([0,2 \pi))=I$, then there exists a spectral family $E$ on a larger Hilbert space $H^{\prime}$ such that $F=P E P$ where $P$ is the orthogonal projection of $H^{\prime}$ on $H$. This implies that bounded pointwise convergence of a sequence of Borel functions $f_{n}$ to $f$ ensures convergence in the strong operator topology of the corresponding operators $T_{n}=\int f_{n}\left(e^{i \theta}\right) d F$ to $T=\int f\left(e^{i \theta}\right) d F$. With this observation the proof of Theorem 3 also yields the following result.

THEOREM 3A. Let $F$ be a positive operator measure on $[0,2 \pi)$ such that $F([0,2 \pi))$ $=I$. Then the following relation holds for every Borel set $B$ whose boundary contains only points outside $Q$, convergence being taken in the strong operator topology:

$$
F \circ \tau_{k}(B) \stackrel{c}{\longrightarrow} l(B)(I-F(Q))+\sum_{s=1}^{\infty} l_{s}(B) F\left(B_{s}\right) .
$$

As our final theorem of this section we obtain a form of Theorem 3 which characterizes "strongly mixing" spectral measures.

THEOREM 4. Let E be a spectral measure on $[0,2 \pi)$ having as its values projections in a separable Hilbert space $H$. In order that $E$ be the spectral measure of a unitary operator $U$ which is strongly mixing in the sense

$$
U^{k} \rightarrow 0 \text { weakly as } k \rightarrow \infty \text {, }
$$


it is necessary and sufficient that $E$ satisfy the following condition, with convergence in the weak topology:

$$
E \circ \tau_{k}(J) \rightarrow l(J) I \text { for all intervals } J \subset[0,2 \pi) .
$$

Proof. We recall that the formula $U^{k m}=\int e^{i k m \theta} d E \rightarrow 0, m \neq 0$, can be rewritten as

$$
U^{k m}=\int e^{i m \theta} d E \circ \tau_{k} \rightarrow 0, \quad m \neq 0 .
$$

Then by Theorem 2 we may deduce that there exists an $F_{0}$ such that

$$
E \circ \tau_{k}(B) \rightarrow F_{0}(B) \quad \text { for } F_{0} \text {-continuity sets } B,
$$

and

$$
\int e^{i m \theta} d F_{0}=0, \quad m \neq 0, F_{0}([0,2 \pi))=I
$$

This shows that $F_{0}=l I$ and hence that all subintervals of $[0,2 \pi)$ are $F_{0}$-continuity sets, so that (30) holds. The converse is along the lines of Theorem 2 and will be omitted.

4. Applications to complex measures. In this section we apply the theorems of the preceding sections to obtain several results concerning complex measures on a bounded interval and their Fourier-Stieltjes coefficients. As before we normalize to the consideration of complex measures on $[0,2 \pi)$. The various results are based on the simple observation that each such measure $\nu$ can be realized as a spectral type for some spectral resolution $E$ on $[0,2 \pi)$, i.e. $d \nu=d(E x, y)$ for a suitable pair of elements $x$ and $y$. It should also be noted that in most instances the results obtained here via $\$ 3$ may be verified in a direct analytic way.

Throughout, if $\mu, \nu$, etc. are complex measures on $[0,2 \pi)$ then we shall denote their Fourier-Stieltjes coefficients by $\hat{\mu}(m), \hat{v}(m)$, etc., $m=0, \pm 1, \pm 2, \ldots$ We recall the definition of the sets $B_{s}: B_{1}=\{0\}, B_{s}=\{2 \pi j / s: 0<j<s,(j, s)=1\}, s>1$.

Proposition 1. Given a complex measure $\mu$ let $\left\{\mu \circ \tau_{k}\right\}_{k \geqq 1}$ be defined as before. Then

$$
\mu \circ \tau_{k}(B) \stackrel{c}{\longrightarrow} \mu([0,2 \pi)-Q) l(B)+\sum_{s=1}^{\infty} \mu\left(B_{s}\right) l_{s}(B)
$$

for every Borel set $B \subset[0,2 \pi)$ whose boundary is in $[0,2 \pi)-Q$.

This result follows at once from Theorem 3 by applying our observation regarding spectral types. By restating matters in terms of Fourier-Stieltjes coefficients we obtain a different formulation. This utilizes the easily verified fact that

$$
\mu \circ \tau_{\hat{k}}(m)=\hat{\mu}(\mathrm{km}), \quad m=0, \pm 1, \pm 2, \ldots ; k=1,2, \ldots
$$

(An alternate proof would follow directly from the von Neumann ergodic theorem.) 
Proposition 1A. Given a complex measure $\mu$,

$$
\hat{\mu}(k m) \stackrel{c}{\longrightarrow} \hat{v}(m), \quad m=0, \pm 1, \pm 2, \ldots,
$$

where $\nu$ is the limiting measure in Proposition 1.

Now suppose $U$ is a unitary operator which is strongly mixing. Then for any complex measure $\nu$ which is a spectral type for $U$, i.e. $d \nu=d(E x, y)$, one has by (29) the property

$$
\hat{v}(m)=\left(U^{m} x, y\right) \rightarrow 0 \quad \text { as }|m| \rightarrow \infty .
$$

That is, this property holds for every measure $v$ absolutely continuous with respect to a maximal spectral type for $U$ [1]. We shall write $\nu \ll \mu$ to denote absolute continuity.

The remark above suggests that whenever $\mu$ satisfies $\hat{\mu}(m) \rightarrow 0$ and $\nu \ll \mu$, then $\hat{v}(m) \rightarrow 0$. This result is actually valid having first been proved in a slightly weaker form, by Rajchmann [10]. It can be regarded as a generalization of the Riemann-Lebesgue lemma.

Proposition 2 (Generalized Riemann-Lebesgue Lemma). If the complex measure $\mu$ satisfies $\hat{\mu}(m) \rightarrow 0,|m| \rightarrow \infty$, then $\nu \ll \mu$ implies $\hat{\nu}(m) \rightarrow 0,|m| \rightarrow \infty$.

Proof. By the Radon-Nikodym theorem [4]

$$
\int e^{i m \theta} \nu(d \theta)=\int e^{i m \theta} f(\theta) \mu(d \theta)
$$

where $f \in L^{1}(|\mu|),|\mu|$ being the total variation measure for $\mu$. Hence using the fact that the finite trigonometric polynomials are dense in $L^{1}(|\mu|)$ we may write

$$
\begin{aligned}
\left|\int e^{i m \theta} \nu(d \theta)\right| & \leqq\left|\int e^{i m \theta} P_{r}(\theta) \mu(d \theta)\right|+\left|\int e^{i m \theta}\left(f(\theta)-P_{r}(\theta)\right) \mu(d \theta)\right| \\
& \leqq\left|\int e^{i m \theta} P_{r}(\theta) \mu(d \theta)\right|+\left\|f-P_{r}\right\|_{L^{1}(|\mu|)},
\end{aligned}
$$

where $P_{r}$ denotes a finite trigonometric polynomial. Since the second term on the right can be made arbitrarily small, and the first term on the right approaches zero with large $m$, the result follows.

Using the above proposition we obtain at once,

Proposition 3. A unitary operator $U$ is strongly mixing if and only if $\hat{\mu}(m) \rightarrow 0$, $|m| \rightarrow \infty$, where $\mu$ is any maximal spectral type for $U$.

We observe next that the following result, which is a consequence of Proposition 3 and Theorem 4, fully characterizes measures for which $\hat{\mu}(m) \rightarrow 0$.

Proposition 4. A complex measure $\mu$ satisfies $\hat{\mu}(m) \rightarrow 0,|m| \rightarrow \infty$, if and only if $\mu$ satisfies $\mu \circ \tau_{n}(J) \rightarrow \mu([0,2 \pi)) l(J)$, for every interval $J \subset[0,2 \pi)$.

If $\mu$ is a probability measure, then we may restate Proposition 4 in an illuminating probabilistic way. Namely, let $Y$ be the random variable associated with $\mu$ and let 
$(n Y)$ be as before the fractional part of the random variable $n Y$ modulo $2 \pi$. Then we have

Proposition 4A. Let $Y$ be a random variable on $[0,2 \pi)$ and $\mu$ be its probability measure. Then $\lim _{|m| \rightarrow \infty} \hat{\mu}(m)=0$ if and only if the distribution of $(n Y)$ converges to normalized Lebesgue measure.

Proposition 4A permits us to exhibit simple examples of singular measures on $[0,2 \pi)$ whose Fourier-Stieltjes coefficients do not tend to zero. For let

$$
\left\{x_{j}: j=1,2, \ldots\right\}
$$

be a stationary stochastic process with $x_{j}$ assuming values $0,1, \ldots, r-1$, where $r \geqq 2$. Let $Y=\sum_{j=1}^{\infty} x_{j} / r^{j}$ and let $\mu$ be the probability measure associated with $Y$. Then it is known that $\mu$ is either Lebesgue measure or a singular measure. But from the stationarity of the process it follows that the distribution of $(n Y)$ is the same as the distribution of $Y$ whenever $n$ is a power of $r$. Thus we have

Proposition 5. Let $\mu$ be as above. Then $\mu$ itself is either Lebesgue measure or else a singular measure. In the latter case $\hat{\mu}(m) \rightarrow 0,|m| \rightarrow \infty$.

Another simple consequence of Proposition 4A is that if $Y$ is a random variable on $[0,2 \pi)$, and we expand $Y=\sum_{j=1}^{\infty} x_{j} / r^{j}$ to various bases $r=2,3, \ldots$, then either $Y$ is uniformly distributed or else some such expansion (indeed some such expansion with $r$ a prime) does not yield a stationary process $\left\{x_{j}: j=1,2, \ldots\right\}$. We exclude here the trivial case $Y=0$ with probability one.

5. General summability methods. In this section we examine the effect of employing modes of convergence generalizing convergence $\stackrel{c}{\rightarrow}$. There will be no difficulty in formulating our results for two-sided sequences as easily as for one-sided sequences, and for definiteness we shall state matters only in the former context. We denote by $Z$ the set of all integers, positive, negative and zero.

Definition. A-convergence. Let $A=\left(a_{j}^{(n)}\right)_{j \in Z, n \geqq 1}$ denote an infinite matrix of complex numbers whose rows are uniformly bounded in $l_{1}(Z)$ :

$$
\left\|a^{(n)}\right\|_{l_{1}(z)} \leqq K \text {. }
$$

We shall say that $A$ is a convergence method provided that the limit

$$
\lim _{n \rightarrow \infty} \sum_{j \in Z} a_{j}^{(n)} e^{i j \alpha}=\varphi(\alpha)
$$

exists for each $\alpha \in[0,2 \pi)$. If, in addition,

$$
\varphi(\alpha)=0, \quad \alpha \neq 0, \quad \varphi(0)=1,
$$

then we shall say that $A$ is a generalized Cesàro method.

Given any convergence method $A$, the symbol $v_{j} \stackrel{A}{\rightarrow} v$ will denote the statement

$$
\sum_{j \in Z} a_{j}^{(n)} v_{j} \rightarrow v, \quad n \rightarrow \infty,
$$


convergence being taken in the linear topological space containing $v$ and the twosided sequence $\left\{v_{j}\right\}$.

It is easily seen that, in the case of a generalized Cesàro method, all the results obtained earlier which employ convergence $\stackrel{c}{\rightarrow}$ also remain valid when convergence $\stackrel{A}{\rightarrow}$ is employed. In fact the same proofs used before apply, mutatis mutandis. The only new features are that Corollaries 1 and 2 now refer to two-sided sequences $\left\{S_{k}\right\}$, and that Theorems 3 and $3 \mathrm{~A}$ utilize $\tau_{k}$ also for $k<0$. Here the definition of $\tau_{k}$ is as follows:

where $A^{\prime}=-A+2 \pi$.

$$
\tau_{k}(A)=\bigcup_{j=0}^{|k|-1}\left(\frac{1}{|k|} A^{\prime}+\frac{2 \pi j}{|k|}\right), \quad k<0,
$$

In the case of a convergence method $A$ which is not a generalized Cesàro method, Theorems 3 and $3 \mathrm{~A}$ are modified. We have instead the following.

THEOREM 3*. Let A be a convergence method with $\varphi$ as the associated limit function, and let $E$ be a spectral measure on $[0,2 \pi)$ whose values are projections on a separable Hilbert space $H$. Then there exists a weakly countably additive positive operator measure $F_{0}$ such that

$$
\int e^{i m \theta} d F_{0}=\int \varphi((m \theta)) d E, \quad m=0, \pm 1, \pm 2, \ldots
$$

and, for each $F_{0}$-continuity set $B, E \circ \tau_{j}(B) \stackrel{A}{\rightarrow} F_{0}(B)$, convergence being in the strong operator topology.

Moreover, if $\varphi$ is continuous then $F_{0}$ is given by the formula $F_{0}=\sum_{k \in Z} c_{k} E \circ \tau_{k}$ where $c_{k}=\int e^{-i k \theta} \varphi(\theta) d \theta$ are the Fourier coefficients of $\varphi$.

Proof. By the definition of $\varphi$ we have, for all $\theta \in[0,2 \pi)$, the relations

$$
e^{i m k \theta} \stackrel{A}{\longrightarrow} \varphi((m \theta)), \quad m=0, \pm 1, \pm 2, \ldots,
$$

the convergence being bounded convergence from the condition (31). Utilizing the spectral calculus for $U=\int e^{i \theta} d E$ we thus obtain

$$
U^{k m}=\int e^{i k m \theta} d E \stackrel{A}{\longrightarrow} C_{m}=\int \varphi((m \theta)) d E,
$$

convergence being in the strong operator topology by the reasoning in Theorem 3.

By Theorem 2, equation (35) ensures the existence of a strongly countably additive measure $F_{0}$ satisfying the first conclusion of the theorem.

To obtain the second conclusion, notice that (34) implies (in the presence of (31)) that for each $\theta$ there exists a measure $\mu_{\theta}$ on $[0,2 \pi)$ such that both

$$
\hat{\mu}_{\theta}(m)=\varphi((m \theta)), \quad m=0, \pm, \pm 2, \ldots,
$$

and

$$
\chi_{B}((k \theta)) \stackrel{A}{\longrightarrow} \mu_{\theta}(B) \text { for all } \mu_{\theta} \text {-continuity sets } B \text {. }
$$


In fact we can identify $\mu_{\theta}$ in the following manner. Define $\varphi_{n}$ to be the function

$$
\varphi_{n}(\theta)=\sum_{j \in Z} a_{j}^{(n)} e^{i j \theta}, \quad n \geqq 1,
$$

which is clearly continuous by virtue of (37). Then by definition $\varphi$ satisfies

$$
\varphi_{n}(\theta) \rightarrow \varphi(\theta), \quad \theta \in[0,2 \pi) .
$$

Now for each fixed $\theta$ it is easily verified that

$$
\varphi_{n}((m \theta))=\hat{\mu}_{\theta, n}(m), \quad m=0, \pm 1, \pm 2, \ldots,
$$

where $\mu_{\theta, n}$ is the discrete measure on $[0,2 \pi)$ given by

$$
\mu_{\theta, n}=\sum a_{j}^{(n)} \delta_{(-j \theta)} .
$$

Clearly the total variation of the $\left\{\mu_{\theta, n}\right\}$ is bounded uniformly in $\theta$ and $n$ by the constant $K$ occurring in equation (31). It follows from (38) and (39) that the measures $\left\{\mu_{\theta}\right\}$, being weak limits of the $\left\{\mu_{\theta, n}\right\}$, are also of uniformly bounded variation.

Finally, we note that (38) ensures that the Fourier coefficients $c_{j}$ of the function $\varphi$ form an absolutely convergent series. In fact by the dominated convergence theorem

$$
c_{j}=\int e^{-i j \theta} \varphi(\theta) d \theta=\lim _{n \rightarrow \infty} \int e^{-i j \theta} \varphi_{n}(\theta) d \theta=\lim _{n \rightarrow \infty} a_{j}^{(n)}, \quad j \in Z,
$$

so that for any $M$

$$
\sum_{-M}^{M}\left|c_{j}\right|=\lim _{n \rightarrow \infty} \sum_{-M}^{M}\left|a_{j}^{(n)}\right| \leqq K .
$$

Therefore if $\varphi$ is continuous then it coincides everywhere with the sum of its Fourier series: $\varphi(\theta)=\sum_{j \in Z} c_{j} e^{i j \theta}, \theta \in[0,2 \pi)$. In this case we have the following formula for the measures $\left\{\mu_{\theta}\right\}$ :

$$
\mu_{\theta}=\sum c_{j} \delta_{(-j \theta)}, \quad \theta \in[0,2 \pi) .
$$

In order to complete our proof we proceed as follows.

$$
\begin{aligned}
\int \varphi_{n}((m \theta)) d E & =\int \sum a_{j}^{(n)} e^{i m j \theta} d E=\int e^{i m \theta} d\left(\sum a_{j}^{(n)} E \circ \tau_{j}\right), \\
\int \varphi((m \theta)) d E & =\int \sum c_{j} e^{i m j \theta} d E=\int e^{i m \theta} d\left(\sum c_{j} E \circ \tau_{j}\right) .
\end{aligned}
$$

Since (39) assures the strong convergence $\int \varphi_{n}((m \theta)) d E \rightarrow \int \varphi((m \theta)) d E$, we deduce that $F_{0}$ and the operator measure $\sum c_{j} E \circ \tau_{j}$ have the same Fourier-Stieltjes coefficients, which completes the proof.

REMARK. The hypothesis that $\varphi$ is continuous is essential, for when $A$ is a generalized Cesàro method, then $\varphi(\theta)=0, \theta \neq 0, \varphi(0)=1$, and therefore all Fourier coefficients of $\varphi$ are zero while $F_{0}$ is not the zero measure. 
An analogous modified form for Theorem $3 \mathrm{~A}$ is available, but we will omit the statement here.

The arguments above imply in particular the following result.

COROLlaRY 3. Every function $\varphi$ which is a pointwise limit everywhere on $[0,2 \pi)$ of a sequence of continuous functions $\left\{\varphi_{n}\right\}$ whose Fourier coefficients are uniformly bounded in $l_{1}(Z)$ has the following property:

(P) $\{\varphi((m \theta))\}_{m \in Z}$ is the Fourier-Stieltjes series of a measure, for each $\theta \in[0,2 \pi)$.

ACKNOWLEDGMENTS. This work was partially supported by the National Science Foundation under Grants GP8290 and GP11697; an earlier version prepared while we were on Sabbatical leave at Technion-Israel Institute of Technology appeared as Technion Preprint Series No. MT-58. The unfailing consideration and stimulating mathematical atmosphere afforded us at the Technion Mathematics Department are herewith gratefully acknowledged.

\section{BIBLIOGRAPHY}

1. N. I. Ahiezer and I. M. Glazmann, The theory of linear operators in Hilbert space, GITTL, Moscow, 1950; German transl., Akademie-Verlag, Berlin, 1954; English transl., Ungar, New York, 1961. MR 13, 358; MR 16, 596.

2. A. D. Aleksandrov, Additive set functions in abstract spaces, Mat. Sb. 8 (50) (1940), 307-348; ibid. 9 (51) (1941), 563-628; ibid. 13 (55) (1943), 169-238. MR 2, 315; MR 3, 207; MR 6, 275.

3. J. Dieudonné, Sur le théorème de Lebesgue-Nikodym. IV, J. Indian Math. Soc. (N. S.) 15 (1951), 77-86. MR 13, 447.

4. N. Dunford and J. T. Schwartz, Linear operators. I: General theory, Pure and Appl. Math., vol. 7, Interscience, New York and London, 1958. MR 22 \#8302.

5. A. Ionescu-Tulcea and C. Ionescu-Tulcea, Topics in the theory of lifting, Ergebnisse der Mathematik und ihrer Grenzegebiete, Band 48, Springer-Verlag, New York, 1969.

6. J. V. Neumann, Algebraische Repräsentanten der Funktionen "bis auf eine Menge vom Masse Null," J. Reine Angew. Math. 161 (1931), 109-115.

7. M. A. NaǏmark, On a representation of additive operator set functions, C. R. (Dokl.) Acad. Sci. URSS 41 (1943), 359-361. MR 6, 71.

8. J. Neveau, Bases mathématiques du calcul des probabilités, Masson, Paris, 1964; English transl., Holden-Day, San Francisco, Calif., 1965. MR 33 \#6659; MR 33 \#6660.

9. K. R. Parthasarathy, Probability measures on metric spaces, Probability and Math. Statist., no. 3, Academic Press, New York, 1967. MR 37 \#2271.

10. A. Rajchman, Une classe de séries trigonométriques qui convergent presque partout vers zéro, Math. Ann. 101 (1929), 686-700.

11. F. Riesz and B. Sz-Nagy, Leçons d'analyse fonctionnelle, 2nd ed., Akad. Kiadó, Budapest, 1953; English transl., Ungar, New York, 1955. MR 15, 132; MR 17, 175.

12. F. Topsøe, Topology and measure, Lecture Notes in Math., vol. 133, Springer-Verlag, New York, 1970. 87106

Department of Mathematics, University of New Mexico, Albuquerque, New Mexico

Department of Mathematics, Carnegie-Mellon University, Pittsburgh, PennsylVANIA 15213 